\title{
A pseudogene of WDR5 is differentially expressed in brain metastatic breast cancer.
}

Shahan Mamoor, $\mathrm{MS}^{1}$

${ }^{1}$ shahanmamoor@gmail.com

East Islip, NY USA

Metastasis to the brain is a clinical problem in patients with breast cancer ${ }^{1-3}$. We mined published microarray data ${ }^{4,5}$ to compare primary and metastatic tumor transcriptomes for the discovery of genes associated with brain metastasis in humans with metastatic breast cancer. We found that a non-coding RNA transcribed from the WDR5 locus (WDR5p), a pseudogene transcript, was among the transcripts whose quantity was most significantly different in the brain metastases of patients with metastatic breast cancer. WDR5p transcript was present at increased quantities in brain metastatic tissues as compared to primary tumors of the breast. Modulation of WDR5p expression may be relevant to the biology by which tumor cells metastasize from the breast to the brain in humans with metastatic breast cancer.

Keywords: breast cancer, metastasis, brain metastasis, central nervous system metastasis, WDR5 pseudogene, WDR5p, LOC401127, systems biology of breast cancer, targeted therapeutics in breast cancer. 
One report described a $34 \%$ incidence of central nervous system metastases in patients

\section{Methods}

We used datasets GSE10893 ${ }^{4}$ and GSE52604 ${ }^{5}$ for this global differential gene expression analysis of brain metastatic breast cancer in conjunction with GEO2R. GSE10893 was generated using Agilent-011521 Human 1A Microarray G4110A technology with $n=11$ primary breast tumors and $n=3$ brain metastases from patients with breast cancer; analysis was performed using platform GPL885. GSE52604 was generated using Agilent-014850 Whole Human Genome Microarray $4 \times 44 \mathrm{~K}$ G4112F with $n=10$ normal breast tissues and $n=35$ brain metastases from patients with breast cancer; analysis was performed using platform GPL6480. The Benjamini and Hochberg method of $p$-value adjustment was used for ranking of differential expression but raw $p$-values were used to assess statistical significance of global differential expression. Log-transformation of data was auto-detected, and the NCBI generated category of platform annotation was used. A statistical test was performed to evaluate whether WDR5p gene expression was significantly different between primary tumors of the breast and brain metastases in humans with breast cancer using a two-tailed t-test.

\section{$\underline{\text { Results }}$}

We performed global comparative transcriptome analysis of metastatic and primary tumor tissues of patients with metastatic breast, as well as normal tissues of the breast using published microarray data ${ }^{4,5}$ to describe the transcriptional landscape of brain metastasis in human breast cancer in an unbiased fashion and for the discovery of novel therapeutic targets.

\section{WDR5p is differentially expressed in the brain metastases of patients with brain metastatic breast cancer.}

Through blind, systems-level analysis of published microarray data ${ }^{4}$, we identified a pseudogene produced at the WDR5 locus (LOC401127), which we term WDR5p, as a differentially expressed transcript in the brain metastatic tissues of humans with breast cancer (Table 1). When sorting each of the genes expressed in brain metastases based on significance of difference as compared to primary tumors of the breast in patients with breast cancer, WDR5p ranked 148 out of 17418 total transcripts (Chart 1), equating to $99.2 \%$ differential expression. 
Differential expression of WDR $5 p$ in the brain metastases of patients with metastatic breast cancer was statistically significant (Chart $1 ; p=5.56 \mathrm{E}-04$ ).

\section{WDR5p is differentially expressed in the lymph node metastases of patients with breast cancer.}

Differential gene expression analyses that utilize primary and metastatic tumor tissues to discover genes associated with metastasis can be challenging due to the relatively heterogenous cellular composition and constitution of the tumor as compared to normal, untransformed (benign) tissues. Thus, to attempt to validate differential transcriptome-wide differential expression of WDR $5 p$ in brain metastases in human metastatic breast cancer, we queried a second microarray dataset ${ }^{5}$, here comparing normal breast tissues rather than primary tumors of the breast to brain metastases. Again, we identified WDR5p as a differentially expressed gene in the brain metastatic tissues of patients with breast cancer (Chart 2). When sorting each of the genes expressed in brain metastases based on significance of difference as compared to normal breast tissues, WDR5p ranked 1024 out of 41093 total transcripts (Chart 2), equating to $97.5 \%$ differential expression. Differential expression of WDR5p in the brain metastases of patients with metastatic breast cancer was statistically significant (Chart $2 ; p=1.94 \mathrm{e}-07$ ). Thus, differential expression of WDR5p, transcriptome-wide, in the brain metastases of women with metastatic breast cancer was conserved across two independent microarray datasets, both when compared to benign and transformed breast tissues. This suggests that WDR $5 \mathrm{p}$ differential expression in human brain metastatic breast cancer is a bona fide biological process.

\section{WDR5p is expressed at higher levels in the brain metastases of patients with metastatic breast cancer.}

We obtained exact mRNA expression levels for WDR5p, in primary tumors of the breast and in brain metastasis of patients with brain metastatic breast cancer to determine direction and statistical significance of change in WDR5p expression in brain metastatic tissues. We observed significantly higher expression of WDR5p in the brain metastases of patients with breast cancer as compared to primary tumors of the breast: WDR5p was expressed at $-0.26 \pm 0.38$ arbitrary units (AU) in primary tumors of the breast, while it was expressed at $0.94 \pm 0.57 \mathrm{AU}$ in brain metastatic tissues (Figure 1). The difference in WDR $5 \mathrm{p}$ mRNA levels between primary tumors of the breast and brain metastatic tissues was statistically significant (Figure $1 ; p=0.000916$ ).

Thus, by mining published microarray data ${ }^{4,5}$ in an unbiased and systematic fashion, we identified an open reading frame, encoded by WDR5p, as among the genes whose expression was most different, transcriptome-wide, in the brain metastases of patients with breast cancer, when compared to primary tumors of the breast and to normal breast tissues; we observed significantly increased expression of WDR5p in brain metastases as compared to primary tumors of the breast.

\section{Discussion}

We provided evidence here that a pseudogene transcribed from the WDR5 locus, LOC401127, which we have termed WDR5p, is among the genes whose expression is most different in the brain metastases of patients with metastatic breast cancer and that WDR5p mRNA is present at significantly increased quantity in brain metastatic tissues as compared to primary 


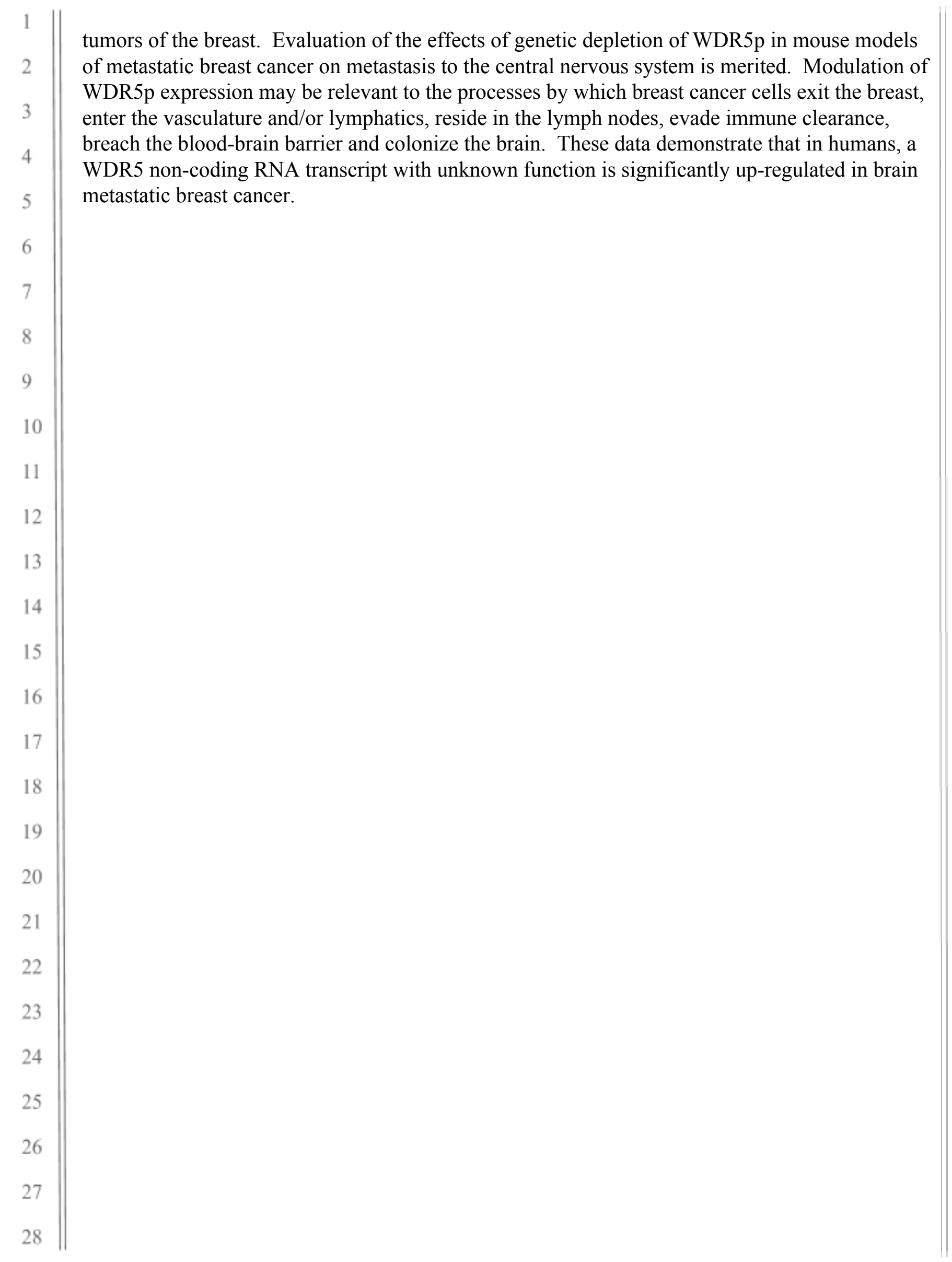




\section{References}

1. Lin, N.U., Amiri-Kordestani, L., Palmieri, D., Liewehr, D.J. and Steeg, P.S., 2013. CNS metastases in breast cancer: old challenge, new frontiers.

2. Bendell, J.C., Domchek, S.M., Burstein, H.J., Harris, L., Younger, J., Kuter, I., Bunnell, C., Rue, M., Gelman, R. and Winer, E., 2003. Central nervous system metastases in women who receive trastuzumab-based therapy for metastatic breast carcinoma. Cancer, 97(12), pp.2972-2977.

3. Tsukada, Y., Fouad, A., Pickren, J.W. and Lane, W.W., 1983. Central nervous system metastasis from breast carcinoma autopsy study. Cancer, 52(12), pp.2349-2354.

4. Weigman, V.J., Chao, H.H., Shabalin, A.A., He, X., Parker, J.S., Nordgard, S.H., Grushko, T., Huo, D., Nwachukwu, C., Nobel, A. and Kristensen, V.N., 2012. Basal-like Breast cancer DNA copy number losses identify genes involved in genomic instability, response to therapy, and patient survival. Breast cancer research and treatment, 133(3), pp.865-880.

5. Sinn, B.V., Fu, C., Lau, R., Litton, J., Tsai, T.H., Murthy, R., Tam, A., Andreopoulou, E., Gong, Y., Murthy, R. and Gould, R., 2019. SET ER/PR: a robust 18-gene predictor for sensitivity to endocrine therapy for metastatic breast cancer. NPJ breast cancer, 5(1), pp.1-8.

6. Awada, A., Colomer, R., Inoue, K., Bondarenko, I., Badwe, R.A., Demetriou, G., Lee, S.C., Mehta, A.O., Kim, S.B., Bachelot, T. and Goswami, C., 2016. Neratinib plus paclitaxel vs trastuzumab plus paclitaxel in previously untreated metastatic ERBB2-positive breast cancer: the NEfERT-T randomized clinical trial. JAMA oncology, 2(12), pp.1557-1564.

7. Györffy, B., Lanczky, A., Eklund, A.C., Denkert, C., Budczies, J., Li, Q. and Szallasi, Z., 2010. An online survival analysis tool to rapidly assess the effect of 22,277 genes on breast cancer prognosis using microarray data of 1,809 patients. Breast cancer research and treatment, 123(3), pp.725-731. 


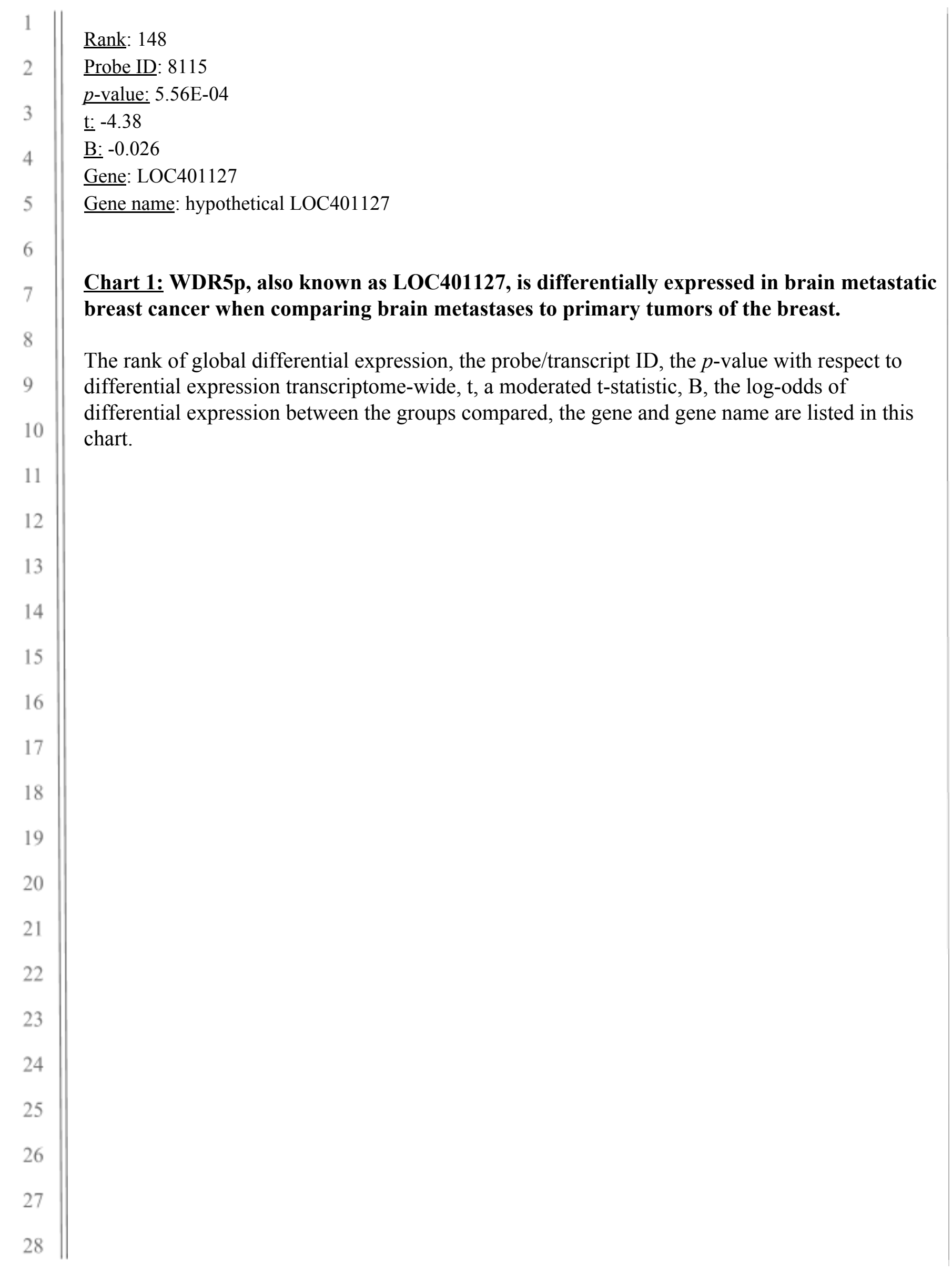




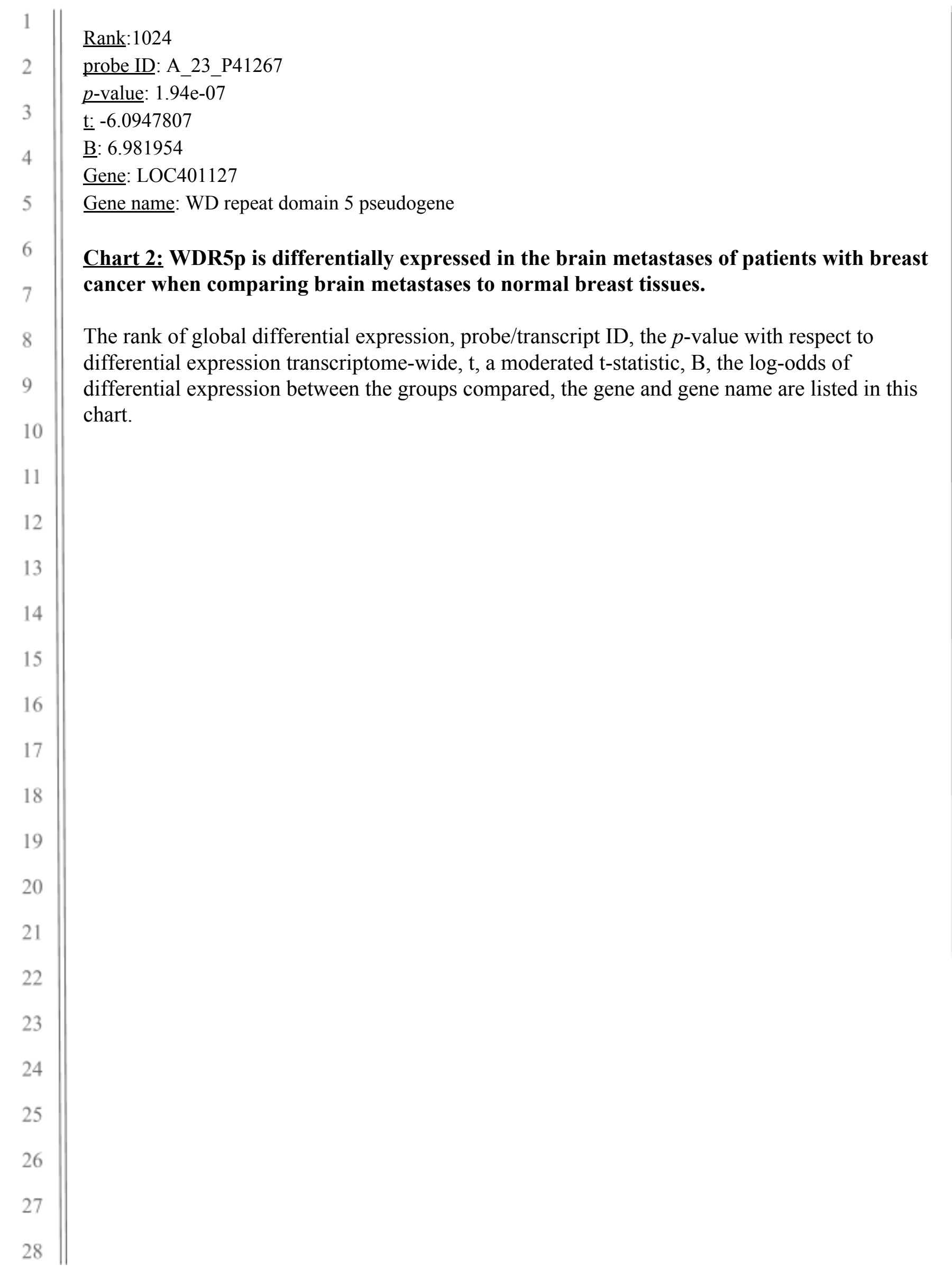




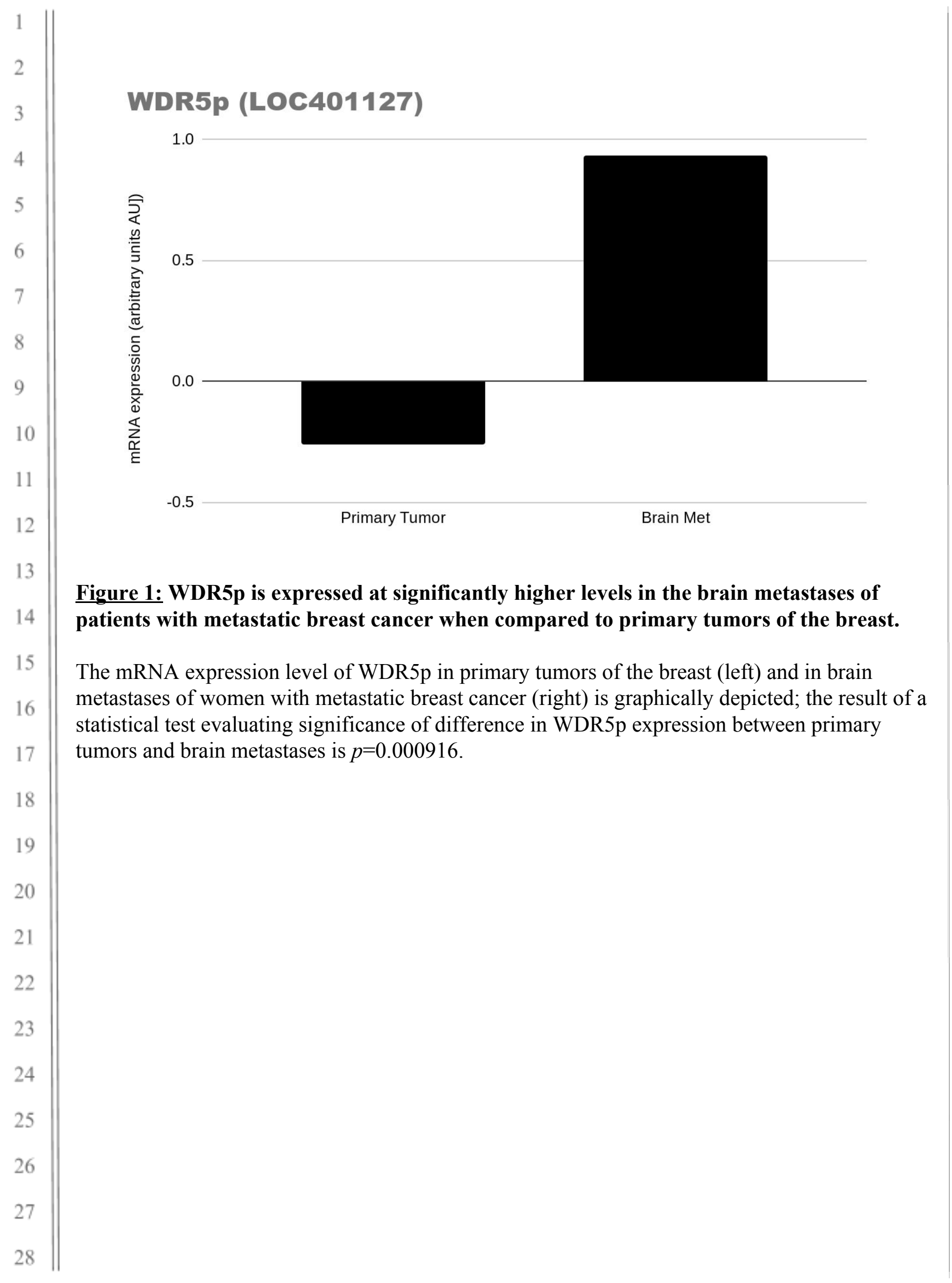

